\title{
Análisis de fracasos en el lanzamiento de nuevos productos en la industria de bebidas en el Perú a partir del modelo stage-gate
}

\section{Analysis of failures in the launch of new products in the beverage industry in Peru from the stage-gate model}

Miluska Alvarado Alarcón

malvaradoa@pucp.edu.pe

Vanessa Alegre Valdivia

vanessa.alegre@pucp.pe

Tatiana Martínez Utía

t.martinez@pucp.pe

Jean Pierre Seclen Luna

iseclen@pucp.pe

Pontificia Universidad Católica del Perú

Este artículo analiza la importancia de estructurar adecuadamente el proceso de desarrollo de nuevos productos (DNP, por sus siglas en inglés). Para ello, identifica algunos fallos en el proceso de DNP en la industria peruana de bebidas a partir de un estudio exploratorio de caso múltiple de nuevos productos que fracasaron en su lanzamiento al mercado. Se empleó una metodología cualitativa para comprender el proceso de DNP, tomando como referencia el modelo stage-gate, uno de los modelos de innovación más sofisticados y difundidos en la práctica actual. El estudio encontró que las empresas estudiadas cometieron graves errores en sus procesos de DNP, tales como no escuchar la voz del consumidor desde el inicio y no realizar pruebas de mercado piloto, entre otros.

Palabras clave: desarrollo de nuevos productos, stage-gate, industria de bebidas y cerveza, fracasos

This article analyses the importance of properly structuring the process of the new products development (NPD). It identifies some failures in the NPD process in the Peruvian beverage industry based on an exploratory study of multiple cases of new products that failed to be launched on the market. A qualitative methodology was used to understand the NPD process, taking as a reference the stage-gate model since it's one of the most sophisticated and disseminated innovation models in current practice. The study founded that the companies analysed made serious mistakes in their NPD processes, such as not listening to the consumer's voice since the beginning of the process and not conducting pilot market tests, among others.

Keywords: new products development, stage-gate, beverage industry, failures 


\section{Introducción}

El desarrollo de nuevos productos (DNP) a lo largo del tiempo ha adquirido una gran importancia debido a su contribución en la cuota de mercado, la facturación y la rentabilidad de las empresas (Fernández, 2009). No obstante, existe una alta probabilidad de fracasar en el lanzamiento de nuevos productos al mercado (Christensen, Cook \& Hall, 2005), lo que implica grandes pérdidas para las empresas (Kuczmarski, 1997). Cooper, Edgett y Kleinschmidt (2002) encontraron en su estudio que muchas empresas exitosas emplean procesos formales de DNP con criterios de decisión bien definidos. Por lo mismo, cabe resaltar que, en la actualidad, existe una gran diversidad de modelos de DNP que pueden estar compuestos de diversas fases y subprocesos, los cuales inician con la generación de ideas y abarcan hasta el lanzamiento de un nuevo producto al mercado (Cooper, 1993).

Uno de los modelos de innovación más sofisticados y difundidos en la práctica es el stage-gate propuesto por Cooper (1993), que consiste en cinco fases secuenciales que están fuertemente orientadas al consumidor (Sattler, 2011; Seclen \& Barrutia, 2019). E stage-gate divide el proceso de innovación en cinco etapas: a) investigación preliminar, que es una rápida exploración del proyecto; b) investigación en profundidad, que tiene como objetivo armar una estrategia previa al desarrollo e incluye un estudio de las necesidades del consumidor, la información técnica y la proyección financiera del proyecto; c) desarrollo, que implica la elaboración del prototipo, la planificación y diseño del proceso de fabricación a realizar; d) las pruebas y la validación, que tienen por propósito verificar la propuesta del nuevo producto mediante exámenes internos, pruebas de campo, pilotos y pruebas de ventas reales; y e) el lanzamiento, que concierne a la comercialización en general del nuevo producto (Fernández, 2009).

Nuestro estudio analiza la importancia de estructurar adecuadamente el proceso de DNP para el éxito comercial (Cooper, 2008). Otra contribución de este estudio es que identificamos algunos fallos en el proceso de DNP en la industria peruana de bebidas de la última década, tomando como punto de partida el modelo stage-gate. En particular, se demuestra que los procesos de DNP de las empresas analizadas, a pesar de que se realizan de manera independiente, presentan algunos aciertos y errores en común (Alvarado, Alegre, Martínez \& Seclen, 2017).

Actualmente, no existen cifras en estudios o informes oficiales sobre la cantidad de fracasos de nuevos productos en el Perú, por lo que la temática adquiere especial relevancia. En particular, es importante comprender todo el proceso de DNP y conocer cuáles son las fallas cometidas en el mismo.

Este artículo primero presenta un marco teórico sobre la importancia del proceso de DNP y del modelo stage-gate. En segundo lugar, sustenta el trabajo empírico a partir de entrevistas hechas a profundidad y sus respectivos hallazgos. Por último, interpreta el proceso de DNP de las tres empresas analizadas. 


\section{Marco teórico}

\subsection{Desarrollo de nuevos productos y fracasos en su lanzamiento}

Tradicionalmente, la literatura sobre la innovación de productos se ha centrado sobre el desarrollo de nuevos productos (DNP). Diversos autores han explorado una variedad de temas relacionados con el DNP. Por ejemplo, Brown y Eisenhardt (1995) realizaron una amplia revisión de la literatura y encontraron que muchos estudios estaban orientados al ámbito organizacional para desarrollar proyectos y realizar ingeniería recurrente. Asimismo, Krishnan y Ulrich (2001) se enfocaron fundamentalmente en el marketing, la gestión organizacional, los proyectos de ingeniería y la gestión de operaciones. Hansen y Birkinshaw (2007), por su lado, analizaron la cadena de valor de la innovación para concluir que el DNP es una de sus actividades clave.

Ahora, de acuerdo a cómo se gestione el proceso del DNP, existe una alta o baja probabilidad de fracasar en el lanzamiento del nuevo producto. Hoy en día, más de 30000 nuevos productos de consumo son lanzados al mercado cada año; sin embargo, más del 90\% de ellos fracasa (Christensen et al., 2005). Incluso las compañías más exitosas fracasan en un $35 \%$ de los lanzamientos de sus nuevos productos al mercado, lo que implica grandes pérdidas para ellas (Kuczmarski, 1997). Por lo tanto, debido a que existe una alta tasa de fracasos, es necesario establecer formalmente procesos estructurados de innovación en el DNP (Fernández, 2009). De hecho, Cooper et al. (2002) encontraron que muchas empresas exitosas emplean procesos formales de DNP con criterios de decisión bien definidos.

Cooper (1993) describe el proceso de innovación de productos mediante su reconocido modelo, denominado stage-gate, que consiste en cinco fases secuenciales que están fuertemente orientadas al consumidor. Este modelo de innovación es uno de los más sofisticados y difundidos en la práctica (Sattler, 2011; Seclen, 2019), por lo que, para los fines de esta investigación, lo tomaremos como referencia en nuestro análisis.

\subsection{Modelo stage-gate}

El modelo propuesto por Cooper representa una guía sistematizada y formalizada que abarca desde la aparición de una idea de producto hasta su lanzamiento al mercado (Seclen, 2017). Fue desarrollado en la última década del siglo anterior y ha experimentado varias actualizaciones, las mismas que permiten una mejor adaptación a las necesidades del mercado y de cada empresa (Fernández, 2009). El stage-gate es una alternativa de solución a los esfuerzos de desarrollo de nuevos productos de muchas compañías. Al afrontar una creciente presión para reducir el periodo de tiempo y mejorar los índices de éxito en el lanzamiento de nuevos productos, las empresas buscan implementar procesos para gestionar, dirigir y acelerar sus esfuerzos de innovación. De hecho, son pocas las empresas que presentan altas tasas de éxito en sus procesos de innovación, y por lo general estas han venido implementando este modelo (Cooper, 2011). A continuación, se revisa de forma sucinta las características del modelo stage-gate (ver gráfico 1). 


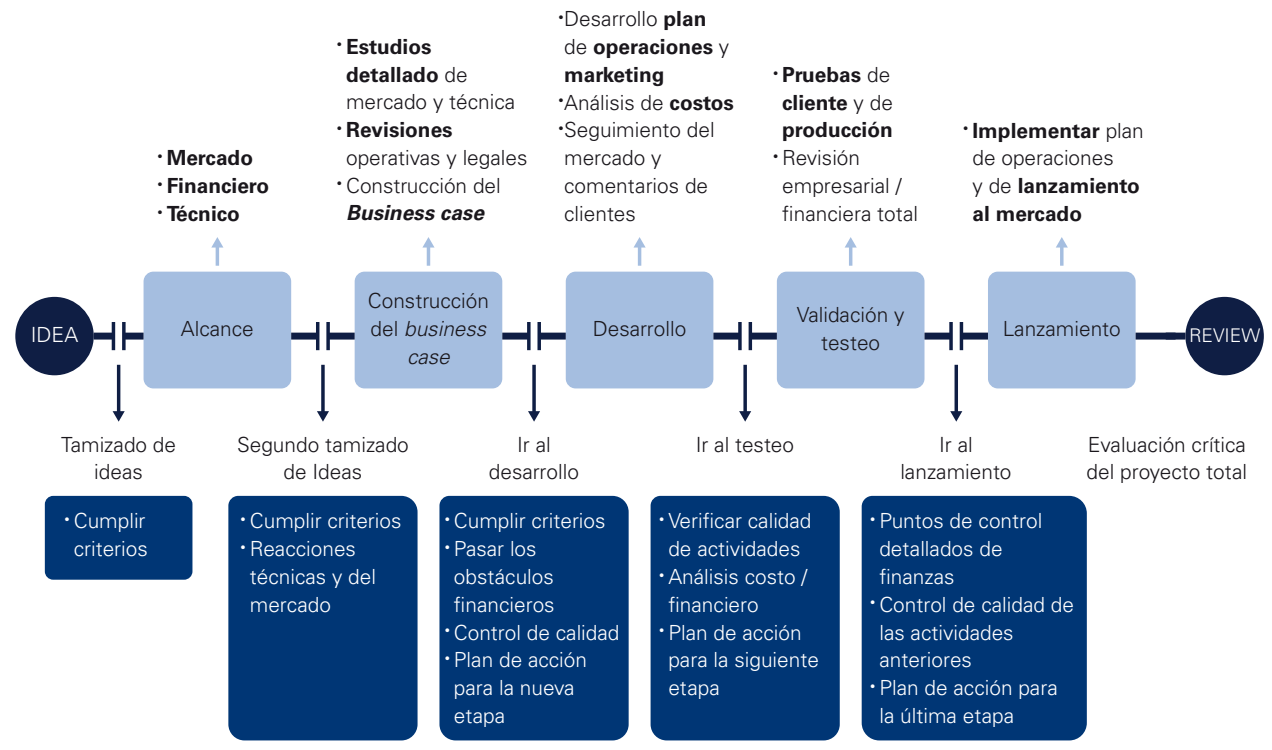

Fuente: Cooper (2011).

\subsubsection{Generación de ideas}

El sistema stage-gate se inicia con el descubrimiento o ideación, que es un momento previo a las etapas del modelo, aunque en algunas empresas se considera como la primera etapa formal debido a la importancia que se le asigna. Las ideas son la materia prima del proceso, y pueden iniciar o finalizar el sistema. Además, deben partir de las necesidades del consumidor. Por lo tanto, una de las tareas más importantes que tienen las compañías es identificar las fuentes potenciales de ideas.

En esta fase, el tamizado de ideas representa el gate 1, que constituye la primera decisión sobre la asignación de recursos de manera global al proyecto. Este criterio - la asignación de recursos- debe cumplirse y encajar con las políticas y estrategias de la empresa, la viabilidad del proyecto y el atractivo del mercado (Cooper, 2011).

\subsubsection{Alcance}

El principal propósito de esta fase es realizar un rápido análisis del proyecto a partir de tres evaluaciones preliminares. De esta forma, en primer lugar, se pretende establecer un alcance superficial del mercado prospecto para el producto; es decir, estimar el atractivo, la aceptabilidad del producto, los competidores y la forma del diseño preliminar. En segundo lugar, el alcance técnico es relevante y consiste en medir la viabilidad del producto, las cuestiones de propiedad intelectual y el cumplimiento de los objetivos de rendimiento desde una perspectiva técnica. En tercer lugar, la dimensión financiera es tomada en cuenta, ya que se estiman las ventas, los costos e inversión requerida para el desarrollo del producto. Estas investigaciones preliminares se complementan para poder realizar recomendaciones y planes para el siguiente filtro. En ese sentido, el segundo tamizado de 
ideas es el gate 2, donde el proyecto está sujeto a una lista de preguntas sobre el estado de preparación y una serie de criterios claves que deben cumplirse, similares a los del gate 1 con mayor profundidad (Cooper, 2011).

\subsubsection{Construcción del business case}

Abarca una investigación detallada que forma parte del proceso en el cual se define de manera clara el producto y se verifica el atractivo del proyecto antes de invertir más recursos. También se revisan los estudios de mercado previos acerca de las necesidades, deseos y preferencias del consumidor. Esta segunda fase da como resultado el "caso de estudio" del proyecto, que incluye la definición integrada del producto, la justificación del proyecto y un detallado plan de acción. En esta fase, pasar al desarrollo es el gate 3, que implica una revisión de todas las actividades realizadas en el paso previo. En esta etapa es muy importante tener los resultados del análisis financiero. Si la decisión es positiva, se revisan los planes de marketing y operaciones, y de ser el caso, estos se aprueban también en esta etapa. Además, se asignan de manera formal los recursos y el equipo completo que estará a cargo (Cooper, 2011).

\subsubsection{Desarrollo}

Es la actividad más visible, ya que se trata del desarrollo físico o prototipo del producto. E equipo del proyecto, especialmente el personal técnico, procede a desarrollar el producto de acuerdo con el plan de acción. De esta forma, se realizan los test internos para asegurar un desempeño adecuado bajo condiciones controladas. Como se puede intuir, en esta fase se pone especial énfasis en aspectos técnicos, aunque las actividades de marketing y operaciones se realizan a la par, pues identificar los lugares donde están los clientes es clave para realizar los testeos del gate 4; de hecho, la atención se enfoca en revisar el progreso y evaluar atractivo del producto y el proyecto. En este gate, el análisis financiero vuelve a tomar importancia, pero ahora basado en data actualizada y más acertada (Cooper, 2011).

\subsubsection{Validación y testeo}

Esta fase se basa en pruebas y validaciones tales como las pruebas internas del producto (usuarios y preferencias), pruebas de producción limitada (mercado o prueba piloto) y la revisión del negocio y el análisis financiero. El objetivo de esta fase es poder dar una validación final para la producción y marketing; es decir, se busca que el producto no solo funcione correctamente en el departamento de I+D, sino también con el cliente. Por lo tanto, el objetivo final es determinar cómo y por qué el cliente responde al producto. El lanzamiento de una prueba piloto cobra especial importancia en mercados con alto grado de incertidumbre pues ayuda a determinar y verificar el pronóstico de ventas; asimismo, se pueden evaluar dos o más alternativas de lanzamiento para ver cuál tiene mejor desempeño.

Ir al lanzamiento es el gate 5, que es la última instancia, encargada de abrir el pase a la comercialización. Aquí se revisan de manera definitiva los planes de marketing y 
operaciones, y se aprueba su ejecución. Si el mercado de prueba tiene un bajo desempeño, el proyecto puede ser descartado (Cooper, 2011).

\subsubsection{El lanzamiento}

Implica la implementación de los planes de operaciones y marketing. Por un lado, el equipo de producción ya está comprado, instalado y a la espera de empezar la producción del producto. Por otro, el plan de marketing es una guía de acción para la introducción de nuevos productos. Consta principalmente de tres partes: objetivos de marketing, estrategias de marketing y los programas de marketing. Respecto a las decisiones de venta y distribución, se deben tomar en cuenta la motivación e incentivos de la fuerza de ventas, ya que el "marketing interno» asegura que se apoye el proyecto. Asimismo, se destaca que en algunos productos el uso de la fuerza de ventas o los canales de distribución existentes puede ser inapropiado. Finalmente, aunque el modelo acaba en el stage 5, después de la comercialización se debe realizar una evaluación que compare los resultados esperados con los reales "postlanzamiento», la cual también debería evaluar las debilidades y fortalezas que se presentaron durante el proyecto para aprender de la experiencia y afinar el proceso. Es con esta revisión que el proyecto se da por finiquitado (Cooper, 2011).

\section{Metodología}

\subsection{Estrategia de investigación}

Este artículo pretende obtener una visión más profunda de las actividades del proceso de desarrollo de nuevos productos, por lo que la adopción de una estrategia de investigación cualitativa es adecuada para este estudio (Saunders, Lewis \& Thornhill, 2009). Para esta investigación se emplea un estudio exploratorio de caso múltiple, ya que es generalmente considerado como una herramienta más robusta que el estudio de caso simple (Yin, 2003). Además, cabe resaltar que es una temática aún poco estudiada y estructurada en el Perú (Alvarado et al., 2017). Por lo mismo, analizamos el proceso del DNP de cuatro nuevos productos de la industria de bebidas gaseosas y cervezas que fracasaron en su lanzamiento al mercado.

\subsection{Selección de los casos}

Se utilizó un enfoque de muestreo teórico para la selección de casos, de acuerdo con el método de investigación cualitativa (Bryman, 2012; Brockman, Rawlston, Jones \& Halstead, 2010; Eisenhardt \& Graebner, 2007). Los casos fueron seleccionados por conveniencia en base a los siguientes criterios:

- Empresas líderes en la industria de bebidas gaseosas y cervezas en el Perú.

- Deben tener procesos de innovación o DNP estructurados.

- Deben haber tenido experiencia de fracaso en el lanzamiento de nuevos productos. 
- Deben tener disposición de compartir información importante y participar del estudio.

Se seleccionó a tres empresas con sede en Lima y se analizaron cuatro casos (ver tabla 1). El caso X1 fue desarrollado por la corporación "Alfa», líder en el mercado de gaseosas con una participación del 67\% y ventas mayores a 300 millones de cajas unitarias. El mayor porcentaje de sus ingresos proviene de las ventas de gaseosas $(71,1 \%)$, seguida por la venta de agua $(21,8 \%)$. El caso Y1 fue desarrollado por la empresa "Beta», que es considerada líder del mercado con una participación de más del 90\%. Dentro de su portafolio de productos se encuentran dos marcas que tienen juntas obtienen un 64\% del mercado de cervezas. Por último, los casos Z1 y Z2 fueron lanzados por la empresa "Charly", que ha tenido éxito en mercados como los de bebidas carbonatadas, aguas embotelladas y bebidas deportivas, pero que en el 2007 decidió incursionar en la industria de la cerveza (Euromonitor, 2016a, 2016b).

Tabla 1. Casos de estudio

\begin{tabular}{c|c|c} 
caso & producto & empresa \\
\hline Caso X1 & Gaseosa & Alfa \\
\hline Caso Y1 & Cerveza & Beta \\
\hline Caso Z1 & Cerveza & Charly \\
\hline Caso Z2 & Cerveza & Charly
\end{tabular}

Fuente: elaboración propia.

La variedad de casos en la industria evaluada proporcionó un gran conjunto de datos que, en última instancia, aumentó la validez y precisión de esta investigación cualitativa (Jack \& Raturi, 2006; Saunders et al., 2009). Las empresas se identifican como Alfa, Bravo y Charly, para mantener su anonimato.

\subsection{Recolección de datos}

El levantamiento de la información se realizó de abril a julio del 2017 y fue llevado a cabo por los autores a través de entrevistas personales con los ejecutivos encargados del proceso de DNP de los respectivos casos, así como con expertos del ámbito académico y de la industria de bebidas gaseosas y cervezas para profundizar en el entendimiento del sector (ver tabla 2). También se realizó una búsqueda exhaustiva de información en la página web oficial de las empresas y se utilizaron documentos internos (por ejemplo, informes anuales) para complementar los datos de las entrevistas, así como mejorar la fiabilidad y la triangulación de datos (Jack \& Raturi, 2006). Los datos de las entrevistas se devolvieron a los entrevistados para la aclaración de las citas y la confirmación de su exactitud (Oliva \& Watson, 2011). 
Tabla 2. Lista de entrevistados

\begin{tabular}{|c|c|c|c|}
\hline código & cargo & fecha & duración \\
\hline E1 & Profesor de Marketing en la PUCP & $27 / 04 / 2017$ & 32 minutos \\
\hline E2 & Jefe de Marca Pepsico & 02/05/2017 & 35 minutos \\
\hline E3 & Jefe de Desarrollo de Categoría de Alfa & 03/05/2017 & 42 minutos \\
\hline E4 & Brand Manager de Alfa & 03/05/2017 & 43 minutos \\
\hline E5 & Analista de Trade Marketing de Alfa & 05/05/2017 & 22 minutos \\
\hline E6 & Director de Marcas Nuevas de Bravo & $16 / 05 / 2017$ & 70 minutos \\
\hline E7 & Marketing Manager de Alfa & 23/05/2017 & 70 minutos \\
\hline E8 & Gerente de Desarrollo Comercial de Alfa & $12 / 06 / 2017$ & 47 minutos \\
\hline E9 & Coordinadora de Productos en Bravo & 20/06/2017 & 33 minutos \\
\hline E10 & Gerente Comercial de Cervezas en Charly & $25 / 06 / 2017$ & 43 minutos \\
\hline E11 & Gerente Regional de Marketing en Charly & 03/07/2017 & 49 minutos \\
\hline E12 & Director de Maestría de Marketing en ESAN & 03/07/2017 & 45 minutos \\
\hline E13 & Profesor de Marketing en la Universidad de Lima & 07/07/2017 & 50 minutos \\
\hline E14 & Brand Manager de Cervecerías en Bravo & $10 / 07 / 2017$ & 60 minutos \\
\hline E15 & Gerente de Marketing de Alicorp & $12 / 07 / 2017$ & 80 minutos \\
\hline E16 & Gerente General de Consultoría y Enfoque & $12 / 07 / 2017$ & 56 minutos \\
\hline E17 & Profesor de Innovación y Tecnología en UNAM & $12 / 07 / 2017$ & 110 minutos \\
\hline
\end{tabular}

Fuente: elaboración propia.

\subsection{Proceso de análisis de la información}

Se adoptó un método de análisis de datos en dos pasos (Beverland \& Lindgreen, 2010). En primer lugar, cada caso se examinó por separado para destacar las actividades únicas en sus procesos de DNP; y, luego, se realizó un análisis cruzado de casos con el propósito de identificar patrones similares para conocer el proceso de DNP que empleó cada empresa.

En el caso de las respuestas incluidas como citas directas de los entrevistados, estas fueron utilizadas para reducir el error de interpretación (Oliva \& Watson, 2011). Finalmente, también se entrevistó a especialistas en la materia, quienes brindaron su opinión. Todo ello contribuyó a construir una visión más crítica y menos sesgada para el análisis y establecimiento de conclusiones en la investigación (Yin, 2003).

\section{Resultados}

En esta sección, se analizarán cada uno de los casos de manera resumida, destacando los principales hallazgos obtenidos. 


\subsection{Caso X1}

Uno de los valores esenciales para esta empresa es la innovación, pues a lo largo del tiempo se ha caracterizado por lanzar al mercado productos nuevos. En esa línea, decidieron extender su marca emblemática en el 2014 con un nuevo sabor a chicha (producto X1). Para este lanzamiento establecieron que la inversión de marketing se dividiría en dos partes. Un 30\% fue destinado a entregar muestras para que el público pudiese probar el nuevo producto, ya que habían creado una gran expectativa previa a la venta. El 70\% restante se utilizó para la comunicación ATL o above the line (Freitas, 2014).

Los principales estudios que ayudaron a la obtención de la idea fueron tres: el primero se centró en las motivaciones del consumidor para elegir un producto; el segundo se centró en el amor a la marca, el posicionamiento, la preferencia y la elasticidad de precio; y, finalmente, el tercer estudio sirvió para conocer las principales ocasiones de consumo de las bebidas. Este último estudio reveló que el $70 \%$ del consumo de gaseosas y chicha morada se da como acompañamiento de las comidas. Además, se dio a conocer la creciente tendencia del consumo de bebidas naturales que, en consecuencia, afectó a la categoría de gaseosas. Por ello, decidieron dinamizar esta última categoría mediante el lanzamiento de un producto que compitiera con los refrescos: una bebida con sabor a chicha morada.

Para poder llegar a la idea del producto X1 utilizamos muchas fuentes de información. Por un lado, un estudio que buscaba identificar las motivaciones detrás de las decisiones de compra (...). También tenemos un estudio que lo que hace es identificar las principales ocasiones de consumo de un producto (...), la principal ocasión de consumo de gaseosas es con las comidas. Por último, nosotros tenemos otro estudio que se llama Be You que (...) es sobre posicionamiento de las marcas; o sea, mide el Brand Love, la identificación, la preferencia, si vale lo que cuesta para ver la elasticidad de precio (E8).

Desde un inicio, se planteó que el nuevo producto debía ser lanzado bajo el respaldo de la marca más fuerte de la empresa (marca de gaseosa líder en el Perú), que será llamada en esta investigación producto X2. Luego de seleccionar la idea de una bebida con sabor a chicha para la categoría de gaseosas, realizaron las investigaciones preliminares a través de consultoras externas.

Se vio que la marca X2 y la chicha morada comparten atributos de marca y generaban orgullo nacional (...). Por estas fuentes de investigación vimos la oportunidad de fusionarlas (...) encontrar una idea creativa de innovación que nos permita fusionar ambas bebidas (...) (E7).

Ese es un mea culpa que tenemos, si haces una extensión de sabor fue ambicioso pensar que ibas a tener todo ese alcance, todos los NSE (niveles socioeconómicos), todos los niveles sociodemográficos (...). Creo que ahí nos faltó medir el riesgo y pensar que la gaseosa X2 se demoró años en meterse en 
el paladar de la gente, se debió pensar que ese sabor debió estar dirigido a un grupo más pequeño (E4).

Respecto a los atributos que caracterizaron al producto, se decidió mantener el nivel de gas de las bebidas carbonatadas. En ese punto, tanto los involucrados en el proceso como los especialistas opinaron que había sido un error usar el sabor a chicha.

Un aprendizaje fue tocar sabores bien sagrados, muy peruanos, muy nuestros. Y obviamente estos sabores más sagrados son de preparaciones más caseras que productos artificiales posicionados a ser gaseosas (...) (E5).

Para continuar con el proceso, los criterios tomados en cuenta para destinar recursos se enfocaron en detectar una oportunidad de negocio clara, viable y realizable. Ya con la idea y las aprobaciones necesarias, el prototipo del producto se fabricó en los centros de innovación de la corporación, ubicados en Brasil, Centroamérica y EE. UU. En él se utilizaron los mejores insumos y se trabajó con diversos proveedores con el objetivo de elaborar el brief del proyecto para enviarlo a Perú.

Luego, se verificó la propuesta en base a test internos. Para esta parte del proceso, los especialistas consideran indispensables los test de sabor, ya que con ellos se pueden corregir aspectos que se obviaron en etapas anteriores. Asimismo, los involucrados en el proceso afirman que sí se realizaron pilotos de producción, pues era necesario probar las líneas, el diseño del proceso de fabricación, definir cómo serían los moldes y diseños, etcétera.

En el caso de las validaciones externas, no se realizó ninguna prueba piloto del producto por temas de confidencialidad, pues se temía que la idea se filtrara y que la competencia pudiera reaccionar antes del lanzamiento. Respecto a ello, los expertos comentan que, de haberse realizado una validación externa, las probabilidades de fracaso se hubieran podido reducir.

Por otro lado, cuando el producto ya estaba listo para ser lanzado, las estrategias de comunicación y comercialización llevadas a cabo fueron las mismas que aplicaron para el producto $X 2$, teniendo como principal objetivo llegar a todo el país. La idea era que donde hubiera un producto X2 estuviera también el producto X1. Inclusive, se aplicó una estrategia que reforzaría la anterior, el bundle pack, que consistía en dar una o dos botellas de producto $\mathrm{X} 2$ por la compra de un paquete del producto X1.

En la comunicación, se promocionó como una bebida nueva con el respaldo de la marca del producto $\mathrm{X} 2$, pues se buscaba que el consumidor la asociara con la calidad y pensara que iba a tener una mejor chicha que la que toma en casa. No obstante, no se llegó a comunicar con claridad que era una bebida gasificada, lo cual ocasionó que se sobrestimaran las expectativas del consumidor. Por esa razón, se decidió cambiar la estrategia de promoción y se empezó a comunicar que la bebida contenía gas.

Yo creo que han tenido bien claro el posicionamiento que tenía este producto, pero no lo bajaron bien a la comunicación, eso sí fue un error y generó expectativas distintas o sobre expectativas cuando se probó (E16). 
Finalmente, después de seis meses de que el producto estuviera en el mercado, la empresa tomó la decisión de dejar de comercializarlo ya que no se logró obtener el pronóstico planificado.

\subsection{CasoY1}

El segundo caso fue un producto propuesto por la empresa Beta, que actualmente es líder del mercado cervecero en el Perú, aunque también está presente en otras categorías como gaseosas, agua embotellada y energizantes. Al formar parte de un holding que tiene casi la totalidad del mercado de cervezas, tiene como nueva ambición el aumento de su penetración y la captura de nuevas ocasiones de consumo (empresa Beta, 2016).

Es en el año 2009 cuando la empresa decide incrementar su cuota de mercado apostando por una nueva cerveza, la cual iba dirigida a un público al que había dejado de lado: el femenino. Este nuevo producto era la cerveza Y1 y fue hecha sobre la base de cebada y sabores cítricos. No contenía el lúpulo, característico de las otras cervezas, y se diferenciaba por tener un bajo nivel de gas y alcohol. Se introdujo al mercado en dos presentaciones: $310 \mathrm{ml}$ y $610 \mathrm{ml}$, con precios sugeridos de $\mathrm{S} / 2,10$ y $S / 3,20$. A pesar de haber tenido una gran inversión (USD 2 millones para el desarrollo y lanzamiento), el producto no gustó al segmento que buscaba atender (empresa Beta, 2009). Según el expresidente de Beta, «El reto en el Perú es que el consumo es compartido. La consumidora ocasional puede tomar este tipo de productos, pero sus acompañantes, generalmente hombres, quieren su cerveza tradicional» (empresa Beta, 2016).

En cuanto al producto $Y 1$, en primer lugar, la idea nació gracias a que la empresa promueve la innovación mediante eventos tipo workshop en donde los trabajadores opinan sobre qué nuevas ideas lanzar. Además, realizaron benchmarks de tendencias mundiales y encontraron que, en países como España, uno de los productos que tiene alto volumen de ventas y margen son las cervezas saborizadas. A raíz de eso, se empezó a estudiar al consumidor peruano y sus costumbres. Pese a ello, los especialistas afirmaron que la empresa se había equivocado en realizar un benchmark con España, ya que este país no refleja la realidad del mercado peruano. Para ellos, la idea debía responder a una necesidad del mercado y cuestionaron que realmente fuera una necesidad del segmento elegido.

Se utilizó templates para saber cómo encontrar una idea innovadora, ya que la clave para desarrollar un nuevo producto es creer que la idea es muy buena. El template para el caso del producto Y1 tenía una hipótesis de estimación de volumen y precio (E6).

A nivel mundial hay muchas cervezas saborizadas, en España es uno de los productos que tienen alto volumen de ventas y margen. A raíz de eso, y de empezar a observar algunas costumbres en el país, nos decidimos (E9).

Ya con la idea del nuevo producto, la empresa decidió realizar estudios para validar la necesidad del mercado. Estos permitieron conocer la situación del mercado, así como las ocasiones y rituales de consumo de las mujeres. 
Lo que se identificó es que había un público femenino que mezcla la cerveza con gaseosa, lo cual es real en los niveles bajos, la mezclan (...) lo que no les gusta es el amargo de la cerveza. A raíz de ello se abre la posibilidad; o sea, teniendo en cuenta la tendencia mundial de cervezas sin sabor amargo y la necesidad local, se opta por lanzar una cerveza frutada (E9).

Con los hallazgos obtenidos por los estudios cuantitativos y cualitativos se pudieron aterrizar los atributos del producto. Los insights obtenidos fueron que las mujeres tenían una receptividad del amargor diferente al de los hombres; asimismo, que a ellas sí les importa cuidar su apariencia y la cerveza las "embotaba" y las hacía ir a los servicios higiénicos con mucha frecuencia. Por ello, decidieron que el líquido debía tener un menor grado de alcohol, no debía raspar la garganta al ser ingerido y tenía que ser menos diurética, con un sabor frutado tipo a champagne. Otro atributo identificado fue el emocional, en el cual la mujer se siente parte del grupo cuando consume cervezas en «cajón».

En Perú se hizo un estudio antes de lanzar el producto, lo cual permitió ver la situación de mercado, las ocasiones de consumo, los rituales de consumo en las mujeres. Esto reveló que había un gran potencial en mujeres de sectores medios y bajos. (...) hubo un error de entendimiento, porque el nivel bajo no tiene poder de decisión. Por el tema de volumen, se optó por tomar como target principal a los sectores más bajos y como target secundario los NSE (niveles socioeconómicos) más altos (E9).

Según los especialistas, el intento de innovar en esta categoría fue erróneo porque con el producto buscaban eliminar el atributo diferenciador de la cerveza: el sabor amargo. Además, opinan que no se logró entender bien qué deseaban las mujeres y, por el contrario, se debió considerar como trasfondo el por qué consumían cervezas.

(...) creo que querían producir un refresco, no una cerveza. Trataron de cambiar la categoría del producto, (...) una de las características principales de la cerveza es el sabor (...). El insumo principal no lo pueden cambiar, que es la cebada. El lúpulo produce amargor, es un ingrediente especial que se usa para dar ciertas características de sabor, aroma inclusive, a la cerveza. (...) El lúpulo que se pone en pocas cantidades aporta mucho al sabor amargo y al aroma de la cerveza. (...) le quitaron un elemento clave y fundamental a la cerveza. Hasta en eso demostraron confusión, o sea lanzan una cerveza al mercado que no es cerveza, que quiere ser más un refresco (...) (E17).

En cuanto al público objetivo, este se dirigió en un primer momento a mujeres de niveles socioeconómicos más bajos y como público secundario a mujeres de niveles socioeconómicos más altos (por el nivel de venta que representaba cada uno). Sin embargo, después del lanzamiento, se buscó que el producto se dirigiera a un público más joven al que no le gustaba la cerveza y que podía considerar a la cerveza Y1 como una buena opción. 
Debido a estos cambios, los entrevistados concluyeron que redirigir el público objetivo fue un gran error, pues generó confusión en los consumidores.

Respecto a los recursos utilizados, la empresa decidió que antes de seguir con el proyecto se realizara una verificación de la oportunidad para elegir la que aporte mayor valor y tenga mayor capacidad de ganar. Es en esta parte del proceso, añade uno de los involucrados, donde se inflaron las expectativas del volumen de ventas que esperaban obtener. Por ello, la inversión que se le destinó al proyecto fue superior al real.

Después de validar los recursos, se pasó a la elaboración de prototipos y test internos. Para elaborar el prototipo se estudió al consumidor, buscando saber qué era lo que esperaban de este tipo de bebidas. Con esta información a la mano, se intentó plasmar las características escogidas en un prototipo.

Un problema fueron las expectativas de volumen, en el estudio, por ejemplo, salió que debía ser cien el volumen y políticamente lo subieron a trescientos, entonces la inversión que le dieron fue de trescientos y eso era un nicho. Y ser nicho no es malo, solamente que no le vas a dar la inversión como a una marca que es una mainstream (...) (E9).

En cuanto al testeo externo, se realizaron diversas pruebas que permitieron validar la propuesta. Una de ellas fue un testeo cuantitativo de sabor llamado «prueba ciega», cuyos resultados arrojaron una aceptación del producto de 20/20. Ante ello, los especialistas indicaron que era probable que todas las variables extrañas no estuvieran $100 \%$ controladas. Los especialistas añadieron que las pruebas de sabor y testeos son confiables si se realizan en conjunto con pruebas estadísticas y geográficamente representativas del nivel socioeconómico, con las cuales se obtiene una obtiene mayor validez de resultados que ayuda a la hora de tomar decisiones. Además, se realizó un piloto de producción con un lote mínimo con el objetivo de poder precisar los costos y conocer cuán rentable sería el producto.

(...) podría ser que no estuvieran $100 \%$ controladas las variables extrañas, según las investigaciones. No debe haber solo una investigación (...). Necesitas de varios estudios (...). Además, tuvieron que hacer un cuantitativo para poder determinar si es viable o no, pero también hay que ver si las marcas resisten más productos. Definitivamente, lo cualitativo no puede ser la única técnica. Las pruebas de sabor y testeos son confiables si las haces estadísticamente (...). Estas pruebas se hacen con pruebas estadísticas y representativos geográficos del nivel socioeconómico para que tenga la validez suficiente y puedas tomar decisiones; si sí o si no. Desde lo cualitativo no puedes hacer esto (E16).

Finalmente, el producto no contó con lanzamiento en un mercado piloto, ya que consideraron que era un producto ganador. Durante los primeros meses las ventas fueron altas gracias a la fuerza de venta, pero luego no hubo rotación. Una de las estrategias aplicadas por la fuerza de ventas fue llevarlo a los puntos de venta como «el nuevo producto de la empresa Beta»; sin embargo, los especialistas consideran que ese aspecto fue 
problemático, pues el consumidor lo asociaba a una cerveza amarga. Como consecuencia, hubo un choque entre lo que se esperaba y lo que se probó.

La fuerza de ventas era fuera de lo común (...). Lograba que el producto esté bien colocado. (...) se llegaba al punto de venta como "el nuevo producto de la empresa Beta», (...) entonces el consumidor pensaba que era amarga. Cuando uno tiene algo en la cabeza y al probarlo es diferente, el choque es muy fuerte (E9).

En la estrategia de comercialización consideraron que las mujeres que compran son de los niveles socioeconómicos $A$ y $B$, y que lo hacen de manera individual. No obstante, en el caso de las mujeres de los niveles socioeconómicos C y D, no se les pregunta qué bebida quieren tomar y, más bien, se acoplan a lo que toman los hombres. Por ello, se considera que hubo una incoherencia entre lo que querían que la gente piense de su producto y cómo lo querían vender; y, por esa razón, la publicidad del producto Y1 no estaba orientada a los niveles socioeconómicos C y D.

(...) primero querían que fuera una cerveza de mujeres y la mujer no compra en cajones. (...) En el C y D no hay diferencia porque a las mujeres no les dices "qué bebidas quieres tomar». (...) Creo que ahí había una incoherencia entre lo que querían que la gente piense de su producto y cómo lo querían vender (E16).

Después de que el producto fuera lanzado al mercado, la empresa decidió contratar otro estudio, el cual reveló que se habían cometido errores en la comunicación y segmentación. Además, no se llegó a los lotes mínimos de producción, por lo que después de dos años el producto fue retirado del mercado. Los especialistas afirman que se debió realizar este estudio previamente y creen que quizás hubo mucha resistencia para no retirar el producto del mercado.

La marca no empieza a caminar bien y se da el lineamiento de hacer un «Female», estudio únicamente orientado a la mujer, en el que se recogen más de 8000 casos. (...) Duró un año en campo, lo que se buscaba era entender a la mujer a nivel de consumidor, porque el mundo de cervezas es muy masculino (...). A través de ese estudio se empieza a tomar decisiones en la mitad y final del camino del producto Y1. (...) Este estudio arroja que hay varios tipos de mujeres, más allá del NSE (nivel socioeconómico) y edad, había mujer moderna (vive con las últimas tendencias), la sociable activa (la que organiza), la más recatada, entre otras. Finalmente, lo que se encontró es que a todas las mujeres les gusta la combinación (...) (E9).

\subsection{Casos Z1 y Z2}

La empresa Charly comenzó como una organización familiar y tiene más de 25 años en el mercado peruano. Sus operaciones se iniciaron con la producción de gaseosas en Ayacucho a fines de los años ochenta y su giro de negocio incluye las categorías de bebidas carbonatadas, aguas embotelladas, jugos de fruta, bebidas energizantes y cervezas (empresa Charly, 2016). 
Ocupa el cuarto lugar en volumen de ventas en la categoría de bebidas sin alcohol y el tercer lugar en las carbonatadas en los países en los que opera. En el 2015 vendió más de 3600 millones de litros de bebidas alrededor del mundo y alcanzó una tasa de crecimiento de $22 \%$ entre el año 2000 y 2012. En el año 2007 decidió incursionar en una nueva categoría: las cervezas. Al decidirse a producir estas bebidas, tuvo la necesidad de construir una nueva planta en Huachipa, la cual llegó a tener una capacidad instalada de aproximadamente 800 000 hectolitros anuales. La primera cerveza lanzada fue el producto Z1, que tenía con una propuesta de sabor diferente ya que contaba con una certificación de «Producto de Calidad Premium» emitida por el Bavarian State Institute - Weihenstephan de Múnich, Alemania. Estaba dirigida principalmente para segmentos de ingresos medios (empresa Charly, 2016).

Por otro lado, en el año 2009 se lanzó el producto Z2, una nueva cerveza caracterizada por tener un envase en presentación PET. Su eslogan publicitario era «Tu pasaporte a la diversión». En este caso, esta bebida se dirigió a segmentos de bajos ingresos y a un público joven. Dentro del proceso para la generación de ideas, la empresa Charly se basó en fuentes externas e internas. En cuanto a las externas, se buscó en el mercado cuáles eran las alternativas y marcas que podrían existir, y las oportunidades existentes. En cuanto a las internas, verificaron su capacidad de producción para poder tener un lanzamiento agresivo. Además, los involucrados en el proceso comentaron que la industria de cervezas era madura y que la mayoría del mercado se concentraba en pocos jugadores. Por ello, decidieron escoger un atributo diferenciador como el sabor.

El modelo de negocio de la compañía es ser una empresa desarrolladora de bebidas, no es una empresa de gaseosas, de aguas. Al verlo con esos ojos estás viendo las nuevas oportunidades, actuales o potenciales que pueden ingresar al portafolio del Perú porque aún no se han desarrollado todas las categorías de bebidas que existen. La industria cervecera inicialmente no estuvo pensada para Perú sino para México; sin embargo, en México existió un contexto político distinto que le quitó competitividad (...) eso hizo migrar la decisión a una de las mayores distribuciones, que era Perú. Y en Perú se comienza a desarrollar la cerveza. Ese fue un poco el punto de quiebre de lanzarlo en el Perú. De ahí, viene una segunda marca, que depende del entorno competitivo. En el momento en que la competencia baja los precios y hace una guerra de comunicación, tú lanzas una segunda marca flanker para no debilitar la primera, con otro segmento (E11).

Obtenidas las ideas de los nuevos productos, la compañía realizó estudios para validar la necesidad en el mercado. Para ello, se contó con el apoyo de diversas consultoras que, a través de estudios cuantitativos, obtuvieron información sobre volúmenes, tendencias, formatos en el mercado, etcétera. Por otro lado, también se realizaron varias pruebas cualitativas.

Con todas estas investigaciones se buscó generar una estrategia de diferenciación, ya que se tenía claro que debían ofrecer al consumidor algo distinto. En cuanto a los atributos, fueron diferentes para ambos productos. En el caso del producto Z1, ingresó al mercado con una propuesta distinta de sabor que lo llevó a alcanzar un 10\% de participación en menos de un año. Una de las características más resaltantes es que buscaba ser 
una opción de acompañamiento en las comidas. A pesar de su rápido crecimiento, los entrevistados consideran que fue un error asociarla exclusivamente a las comidas pues en todo el mundo la cerveza es asociada a la diversión.

El producto Z1 entró para la categoría mainstream y entraba con una propuesta de sabor, orientándose hacia los amigos. Por eso utilizamos a Gastón Acurio como imagen; el éxito fue tremendo porque ganamos varios puntos de participación en el mercado en un año (...) entonces (nuestros competidores) comenzaron a bajar precios, a invertir más en medios televisivos (...) Se da una segunda opción y nace el producto Z2, el cual era un cerveza más orientada hacia los jóvenes, hacia la diversión, queríamos que el consumidor tuviera una marca para los jóvenes, que cuando el joven comenzara a tomar cerveza tuviese una marca (E10).

La rápida obtención de participación de mercado provocó que la competencia iniciará estrategias agresivas y, con el afán de proteger la marca, la empresa decidió lanzar el producto Z2 al mercado con el objetivo de que fuese la marca flanker del producto Z1. Ante ello, los expertos opinan que se debió reposicionar al producto Z1 porque, contrariamente a lo que los involucrados pensaron, fue una cerveza con poca presencia en el mercado y sin buen posicionamiento. En cuanto al producto Z2, señalan que estratégicamente se equivocaron al lanzarla como una flanker ya que la marca a la que respaldaba no era fuerte. En cuanto al público objetivo del producto Z1, se buscó competir en la categoría principal y entrar al mercado con una propuesta de sabor orientada hacia los jóvenes, ya que se le quería atribuir elementos como la diversión. Así, la cerveza logró poseer una aceptación importante en corto tiempo.

En el caso del producto Z2, tuvimos la experiencia del lanzamiento del producto Z1, esta era asociada comiendo ceviche, pero no en una fiesta. El error del producto Z1 fue no posicionarla como una marca más activa (...). Desde mi experiencia (...) la cerveza es asociada con la diversión y ahí nos faltó hacer una marca con esa característica, porque ya en ese momento teníamos una importante participación. Por ello, se genera una segunda marca: (el) producto Z2 (...) (E11).

Las validaciones internas se realizaron mediante prototipos y testeos. También, se realizaron pruebas pre y post, como grupos de enfoque y pruebas sensoriales con varias alternativas para poder elegir la mejor opción. Los especialistas, por su parte, consideran que, además del sabor, también debieron realizarse pruebas de comunicación que validen cómo la publicidad estaba vendiendo el concepto. Son muy pocas las personas que logran diferenciar una cerveza de otra, por lo que en una prueba a ciegas las opiniones pueden ser sesgadas.

Una prueba de concepto de un producto alimenticio o bebida tiene que ir de la mano con la degustación; el concepto es el sabor, el envase, el diseño, etcétera. La prueba de concepto te va a decir si continuas o no con el proceso de desarrollo. Dentro del proceso de desarrollo, se tiene que hacer la prueba del producto y, una 
vez que ya estás a punto de lanzar, tienes que hacer la prueba de comunicación, (es decir,) qué tanto tu publicidad está vendiendo el concepto que supuestamente tenías validado (...) (E13).

Las pruebas piloto de producción que se realizaron para los productos tuvieron como objetivo realizar una estructura de costos preliminar, para luego realizar un lote de producción con dos objetivos. El primero era comprobar las condiciones de la línea de producción y los factores de corrección. El segundo fue aplicar una prueba de calidad y análisis microbiológico para ver que el producto cumpliera con los estándares. Al igual que en los productos $\mathrm{X} 1$ e Y1, la empresa Charly decidió que no se llevaría a cabo el lanzamiento en un mercado piloto.

Los involucrados en los proyectos señalan que la categoría de cervezas fue pensada en primer lugar para lanzarse en México. Sin embargo, el contexto político de ese país le quitó competitividad y buscaron otra operación de la compañía que tuviera gran capacidad de distribución: Perú. Otro de los aspectos que destacan en el desarrollo de esta categoría fue el tiempo de introducción.

(...) se construyó la planta en menos de un año y en paralelo se diseñó todo el producto. El proceso es más ágil por la toma de decisiones, la flexibilidad; el equipo marca (...) sabe cuáles son las actividades en las que se tiene que concentrar y qué hay que hacer (...) todas las áreas trabajan en paralelo. (...) La idea es que un lanzamiento lo puedas tener en menos de un año, seis o siete meses como máximo. (...) nosotros somos muy rápidos, para la Empresa Charly los tiempos no son más de seis meses (E11).

Siguiendo esas líneas, los entrevistados admiten que la empresa se caracteriza por ser ágil en la toma de decisiones, flexible durante todo el proceso y por saber cuáles son las actividades en las que se debía concentrar. No obstante, los especialistas indican que la agilidad y rapidez de la empresa en el lanzamiento de nuevos productos es un arma de doble filo. Por un lado, hay decisiones importantes que requieren tiempo para ser analizadas y a veces, por querer ser más rápidos, se comienzan a omitir pasos.

Para lanzar los productos, las estrategias de comercialización y comunicación persiguieron el objetivo de que el consumidor tenga mayor facilidad para acceder al producto (en cuanto a precios y ubicación), y eso se consiguió por tres razones. Primero, por la alta eficiencia operativa que tienen al reducir sus costos de producción. Segundo, al tener una plataforma de consumo mucho más grande que la de los competidores, la empresa logra vender a muchos y su rentabilidad se obtiene gracias al volumen. Tercero, porque la empresa misma desarrolla sus marcas y no tiene que pagar regalías, a diferencia de la competencia. Esta optimización de recursos se traslada al consumidor a través de precios más bajos. Además, se decidió que las estrategias de comercialización de las cervezas se basaran en publicidad ATL y BTL muy activa (participar en eventos, licorerías, etcétera). Sin embargo, existió una complicación cuando se intentó entrar en bodegas, ya que la competencia lo obstaculizó. Los expertos no concuerdan con que las bebidas alcohólicas deban tener una estrategia de precios bajos, incluso indican que cuando vieron 
la comunicación les pareció que era poco aspiracional. Además, agregan que, aunque se quiera llegar a un segmento bajo, en esa categoría se debe ofrecer un cierto nivel de deseabilidad.

Creo que tuvieron un plan de marketing que no consideró los reales drivers de consumo, (...) creo que el beneficio y la ventaja competitiva de ellos como compañía no necesariamente va a funcionar en todas las categorías. (...) Pero en el caso de las bebidas alcohólicas, de vehículos, incluso de perfumes, hay ciertas categorías que sí necesitan tener un valor; tú puedes estar dentro de un rango y ser de un valor un poco menor, pero no puedes salir a un menor precio (E14).

Finalmente, la empresa, al no obtener los resultados esperados, decidió enfocarse en fortalecer el negocio principal; es decir, la categoría de bebidas. Este fracaso tuvo un impacto negativo por el dinero que se invirtió y se perdió, pero también porque implicó que prácticamente se retiraran de la categoría.

El aprendizaje es no irte al masivo sino ir a nichos. Estos nichos son gigantes ya que la industria es súper grande, así obtengas un $5 \%$ del mercado es un montón y en rentabilidad es muchísimo. Para mí es más fácil construir la pirámide de arriba hacia abajo que de abajo hacia arriba, y nosotros queríamos realizarlo como esta última opción; es decir, empezar con lo masivo. (...) Se comenzó en un mercado en el cual había grandes competidores y en menos de un año le quitaste una importante parte del mercado. La decisión de la compañía en ese momento no fue solo concentrarse con tres marcas, sino seguir fortaleciendo el negocio principal. (...) Nosotros tenemos dos industrias, industria cervecera y la de bebidas, pero el core business de la compañía es la de bebidas; entonces, se tenía que seguir fortaleciendo la industria de bebidas. En ese momento, la empresa se encontraba en una etapa de expansión, de internacionalización (...) y no queríamos distraer los recursos en un mercado complejo (E11).

\section{Discusión, conclusiones e implicancias para el gestor, y limitaciones del estudio}

\subsection{Discusión}

Se ha podido evidenciar que cada empresa parte de un modelo propio y lo "tropicaliza» de acuerdo con sus necesidades. Asimismo, se considera que hay factores internos y externos que se deben tomar en cuenta para el DNP. Desde el punto de vista interno, es importante la comunicación y el trabajo en equipo pues resulta indispensable que todas las áreas conozcan la visión, la misión y los objetivos del nuevo lanzamiento. Desde la perspectiva externa, es relevante encontrar la intersección de las macrotendencias con las preferencias locales para que el producto sea aceptado exitosamente. Para ello, se debe analizar el mercado y evaluar si el momento para el lanzamiento es el adecuado. También se debe realizar pruebas pre y post lanzamiento, ya que permiten saber si el producto satisface una necesidad. 
En cuanto a las tendencias y el estado actual de la industria de bebidas gaseosas y de cervezas en el Perú, en la última década ha habido un cambio, principalmente por tres nuevas características del mercado. Primero, existen nuevas categorías de bebidas. Segundo, se ha ido incrementando el consumo del agua, lo que ha modificado la composición de la categoría de bebidas de acuerdo con los hábitos de consumo. Tercero, ante la alta competencia — que se busca adaptar estos últimos cambios del mercado—, las empresas eligen diferentes estrategias como proteger sus marcas o competir en precios.

En cuanto a las dificultades que tiene la industria, por un lado, en el Perú se visualizan diferentes dinámicas realizadas por las empresas en las categorías según el área geográfica, el nivel socioeconómico y las costumbres. En el caso de la categoría gaseosas, se destaca la influencia del precio en la decisión de compra, ya que existe una gran rivalidad por precios bajos, pues la mayoría de los peruanos no son "leales» a una marca. Sin embargo, es necesario seguir innovando, pues el consumidor peruano es cada vez más exigente y detallista. Por el lado de la categoría de cervezas, un atributo que se destaca es la lealtad que existe hacia las principales marcas. Además, en esta categoría, el consumidor peruano es muy tradicional, aunque está dispuesto a probar diferentes marcas.

\subsection{Conclusiones e implicancias para el gestor}

Este estudio contribuye a la literatura dedicada a estudiar el DNP, ayudando a comprender por qué fracasan las empresas en el lanzamiento de nuevos productos (Fernández, 2009). De acuerdo con el modelo stage-gate, en la fase de ideas se parte del enfoque basado en las necesidades de los clientes; sin embargo, en los casos analizados se ha podido evidenciar que las ideas nacieron como necesidad del negocio en tanto se quería aprovechar las capacidades de las empresas. Asimismo, en la categoría de cervezas, un grave error fue asumir que la necesidad de un país era igual a la de otro. Por lo tanto, se concluye que no se escuchó la voz del consumidor desde el inicio del proceso (Katz, 2004), lo que conllevó a graves errores.

En la fase de alcance, el modelo sugiere que las evaluaciones sirven para determinar la diferencia entre lo que el usuario quiere y necesita. Aquí encontramos que todos los casos realizaron las evaluaciones técnicas, financieras y de mercado. Asimismo, en el business case, encontramos que hubo una definición inadecuada de productos en cuanto a los atributos y el target. Por ejemplo, el producto X1 se enfrentó a un sabor "sagrado", la chicha; y el producto Y1 mostró la ausencia del característico sabor amargo de la cerveza peruana. En cuanto al target, se empleó el masivo para todos los casos y hubo una sobrestimación de la demanda. Por tanto, concluimos que el target es clave en el concepto del producto (Hansen \& Birkinshaw, 2007).

En la fase de desarrollo del producto, el modelo recomienda que se estudie el comportamiento y rendimiento del producto bajo diferentes condiciones. Así, en todos los casos se realizó la validación de los recursos, prototipos y pruebas piloto de producción. No obstante, en el análisis del negocio, el riesgo no fue contemplado para el producto X1 eY1, que inclusive fueron considerados como productos ganadores.

En la fase de validación y testeo, el modelo sugiere que el mercado piloto es de vital importancia cuando hay un alto grado de incertidumbre. Sin embargo, los test que 
se realizaron no fueron integrales y solo se limitaron al sabor. Por ejemplo, en el caso del producto X1, la información fue incompleta y se ignoró el feedback del cliente por miedo al canibalismo; mientras que con el producto Y1 primó la subjetividad en los resultados de sabor. Asimismo, con los productos Z1 y Z2 se creyó un 100\% en la intención de compra. Por estas razones, se concluye que en ninguno de los casos se realizó la prueba de mercado piloto, lo que supone un grave error de proceso.

En la fase de lanzamiento, el modelo recomienda no usar la fuerza de venta existente, así como los canales de distribución, ya que podría inapropiado; sin embargo, en los casos analizados encontramos que se emplearon las mismas estrategias de distribución y la fuerza de ventas. En cuanto al plan de comunicación, encontramos que hubo errores. Por ejemplo, el producto Z2 fue poco aspiracional y en el caso del producto X1 no se comunicaron las características adecuadamente; por lo tanto, la propuesta de valor no fue clara (Cooper, 2011).

A partir de todos estos argumentos, se puede concluir que los principales elementos del proceso de DNP que los gestores deben tomar en cuenta son los siguientes. Primero, es necesario considerar la opinión del consumidor desde el momento inicial del proceso. Segundo, la organización debe estar comprometida en invertir recursos, ya que el proceso de posicionar un producto en el mercado puede durar varios meses. Tercero, se deben impulsar las fuerzas de venta, pues son quienes deben comprar primero la idea. Cuarto, la visibilidad en los puntos de venta, el precio, la comunicación y el diseño deben ser adecuados al estilo de vida del público objetivo.

Por último, este trabajo corrobora empíricamente la necesidad de estructurar adecuadamente los procesos de DNP (Seclen, 2019) y el modelo stage-gate podría ser una buena alternativa a seguir (Ettlie \& Elsenbach, 2007). Esto no significa que se deba seguir un proceso rígido, sino que los procedimientos deben ser flexibles y simplificados ajustados a la realidad de cada empresa (Sethi \& lqbal, 2008). De hecho, la flexibilidad del proceso y la capacidad de respuesta son factores clave de éxito para el DNP en las grandes empresas, especialmente en el caótico entorno de la etapa inicial (lansiti, 1995).

\subsection{Limitaciones del estudio}

Aunque limitada en la capacidad de generalizar los resultados, esta investigación ha subrayado la importancia de estructurar mejor el DNP para su éxito comercial. En definitiva, debido a las limitaciones del estudio, es necesario realizar investigaciones que puedan cuantificar sólidamente el impacto de las prácticas del DNP en el éxito empresarial. Asimismo, en futuros trabajos de investigación sería recomendable incorporar la visión del consumidor final a la hora de explicar por qué fracasan los nuevos productos en su lanzamiento al mercado. 


\section{bibliografía}

Alvarado, M.; V. Alegre, T. Martínez \& J. P. Seclen

2017

¿Por qué fracasan los nuevos productos en su lanzamiento al mercado? Un estudio de casos múltiple en la industria de bebidas en el Perú. Ponencia presentada en II Congreso Internacional de Ciencias de la Gestión. Nuevas tendencia y fronteras de la gestión, 24-27 de octubre. Lima, Perú.

\section{Beverland, M. \& A. Lindgreen}

2010

What makes a good case study?

A positivist review of qualitative

case research. Industrial Marketing

Management, 39(1), 56-63. Recuperado

de: "https://orca.cf.ac.uk/22301/1/

Article\%209.pdf".

Brockman, B. K.;

M. E. Rawlston, M. A. Jones

\& D. Halstead

2010

An exploratory model of interpersonal

cohesiveness in new product development teams. Journal of Product Innovation

Management, 27(2), 201-219.

\section{Brown, S. L. \& K. L. Eisenhardt}

Product development: Past research,

present findings, and future directions.

Academy Management Review, 20(2),

343-378. Recuperado de: «https://doi.

org/10.5465/amr.1995.9507312922".

\section{Bryman, A.}

2012 Social Research Methods. Cuarta edición.

Oxford, Nueva York: Oxford University

Press Inc

\section{Cooper, R. G.}

Winning at New Products: Accelerating the

Process from Idea to Launch. New York:

Addison-Wesley
2008

The stage-gates idea-to-launch processUpdate, what's new, and nexgen systems. Journal of Product Innovation Management, 25(3), 213-232.

2011

Winning at New Products: Creating Value through Innovation. Nueva York: Basic Books.

\section{Cooper, R. G.; S. J. Edgett}

\& E. J. Kleinschmidt

2002

Optimizing the stage-gate process: What best practice companies do. Research Technology Management, 45(5), 21-27. Recuperado de: "https://doi.org/10.1080/08 956308.2002.11671518".

\section{Christensen, C. M.; S. Cook} \& T. Hall

2005

Marketing malpractice: The cause and the cure. Harvard Business Review, 83(12), 74-83. Recuperado de: «https://hbr. org/2005/12/marketing-malpractice-thecause-and-the-cure".

\section{Eisenhardt, K. M.}

\& M. E. Graebner

2007

Theory building from cases:

Opportunities and challenges. Academy

of Management Journal, 50(1), 25-

32. Recuperado de: "https://aom.org/

uploadedFiles/Publications/AMJ/Eisenhart. Graebner.2007.pdf».

\section{Empresa Beta}

2009

Memoria Anual 2009. Recuperado de: «http://Empresa Beta.pe/pdf/MemoriaAnual-2009-Empresa Beta.pdf».

Memoria Anual 2016. Recuperado de: "http://Empresa Beta.pe/pdf/MemoriaAnual-2016-Empresa Beta.pdf". 


\section{bibliografía}

\section{Empresa Charly}

2016

Historia. Recuperado de: «https://

www.empresacharly.com/es/acerca-de-

empresaz/historia/"'.

\section{Ettlie, J. \& J. Elsenbach}

2007

Modified stage-gates regimes in new

product development. Journal of Product

Innovation Management, 24(1), 20-33.

\section{Euromonitor International}

$2016 a$

Beer in Peru. Recuperado de: «http://www. euromonitor.com/beer-in-peru/report».

\section{Euromonitor International}

2016b Carbonates in Peru. Recuperado de: «http://www.euromonitor.com/carbonatesin-peru/report»"

\section{Fernández, H. A. P.}

Innovación y gestión de nuevos productos. una visión estratégica y práctica. Madrid: Pirámide.

\section{Freitas, L.}

2014 Después de 80 años, Marca X lanza un nuevo sabor. Entrevista de Nancy Portugal. Cinta de audio. Lima: Gestión. Recuperado de: «http://gestion.pe/empresas/ despues-80-anos-marca-x-lanza-nuevosabor-2111423".

\section{Hansen, M.T. \& J. Birkinshaw}

The innovation value chain. Harvard Business Review, 85(6), 121130. Recuperado de: "hhttps://hbr.org/2007/06/ the-innovation-value-chain".

\section{lansiti, $\mathbf{M}$.}

development in a turbulent environment. California Management Review, 38(1), 37-58.

Jack, E. P. \& A. S. Raturi

2006

Lessons learned from methodological triangulation in management research. Management Research News, 29(6), 345-357. Recuperado de: "https://doi. org/10.1108/01409170610683833".

\section{Katz, G.}

2004 The voice of the customer. En P. Belliveau, A. Griffin y S. Somermeyer (eds.), The PDMA Toolbook 2 for New Product Development (cap. 7). Hoboken: John Wiley \& Sons

\section{Krishnan, V. \& K. T. Ulrich}

2001 Product development decisions: A review of the literature. Management Science, 47(1), 1-21. Recuperado de: «https://doi. org/10.1287/mnsc.47.1.1.10668"

\section{Kuczmarski, T. D.} Innovación: estrategias de liderazgo para lograr la ventaja competitiva. Bogotá: McGraw-Hill.

\section{Oliva, R. \& N. Watson}

2011 Cross-functional alignment in supply chain planning: A case study of sales and operations planning. Journal of Operations Management, 29(5), 434-448. Recuperado de: «http://www.hbs.edu/faculty/ Publication\%20Files/07-001.pdf».

\section{Sattler, M.}

2011 Excellence in Innovation Management. A Meta-Analytics Review on the Predictors of Innovation Performance. Alemania: Gabler Research. 


\section{bibliografía}

\section{Saunders, M.; P. Lewis}

\& A. Thornhill

2009

Research methods for business students.

Quinta edición. Essex: Pearson.

\section{Seclen, J. P.}

2017

¿Existe relación entre la gestión de la

innovación y los resultados de innovación?

Un análisis exploratorio de empresas

innovadoras peruanas. Ponencia

presentada en la XXVI AEDEM International

Conference. Economy, Business and

Uncertainty: Ideas for an European and

Mediterranean industrial policy?, 4-5

septiembre, Reggio Calabria, Italia.

\section{Seclen, J. P. \& J. Barrutia}

2019

Gestión de la innovación empresarial:

conceptos, modelos y sistemas. Lima:

Fondo Editorial PUCP, In Press.

\section{Seclen, J.P.}

2019

Relationship between innovation process

and innovation results: an exploratory

analysis of innovative Peruvian firms. In

Gil-Lafuente, J. Marino, D. \& Morabito, F. (Eds.) Economy, Business and Uncertainty:

New Ideas for a Euro-Mediterranean

Industrial Policy, (Chapter 14, pp.) Springer

Publishing, In Press.

\section{Sethi, R. \& Z. Iqbal}

2008

Stage-gate controls, learning failure, and adverse effect on novel new products.

Journal of Marketing, 71(1), 118-34.

\section{Yin, R. K.}

Applications of case study research.

Thousand Oaks, California: Sage.

Fecha de recepción: 25/06/2018

Fecha de aceptación: 10/07/2018 